\title{
When all else fails: take a deep breath, meditate, and assume padmasana
}

\author{
Melissa H. Kong ${ }^{1,2} \cdot \operatorname{Roger}$ A. Winkle ${ }^{1,2}$ \\ Received: 4 April 2015 / Accepted: 8 April 2015 / Published online: 13 May 2015 \\ (C) Springer Science+Business Media New York 2015
}

Although common, neurocardiogenic syncope (NCS) encompasses a diversity of clinical presentations, disease states, and underlying pathophysiologic mechanisms. As such, treatment of NCS can prove quite challenging. Numerous treatment options exist for management of NCS including high salt and fluid intake, pharmacologic therapy, tilt training, isometric counterpressure maneuvers, and even implantation of cardiac pacemakers; however, NCS often proves refractory to these measures, for most of which no long-term, controlled clinical evidence of efficacy exists.

A 2011 Cochrane Database meta-analysis examined the effects of pharmacologic and pacemaker therapy on patients with vasovagal or carotid sinus syncope [1]. This metaanalysis included 46 randomized controlled or randomized cross-over trials of drug therapy including beta-blockers, fludrocortisone, alpha-adrenergic agonists, selective serotonin reuptake inhibitors (SSRIs), salt tablets, anticholinergic agents, ACE inhibitors, disopyramide, and dual-chamber pacemaker implantation. The median sample size of these studies was only 42 patients, and the conclusion of the analysis was that insufficient evidence exists to support the use of pharmacologic therapy or pacemaker implants for NCS. Similarly, a more recent and comprehensive meta-analysis comprising both randomized and non-randomized studies further highlights the relative paucity of evidence for the commonly used therapies for vasovagal syncope [2]. When limiting the analysis to randomized studies, only midodrine ( 4 studies with $n=400$, odds

Roger A. Winkle

rawinkle@aol.com

1 Silicon Valley Cardiology, 1950 University Avenue, Suite 160, E. Palo Alto, CA 94303, USA

2 Sequoia Hospital, Redwood City, CA, USA ratio (OR) 0.19 , confidence interval $(\mathrm{CI}) 0.06-0.62, p<0.05)$ and SSRIs ( 2 studies with $n=131$, OR 0.28 , CI $0.01-0.74$, $p<0.05)$ appeared to be effective in preventing recurrent syncope. Meanwhile, beta-blockers, tilt training, and pacemaker implantation failed to show benefit in reducing syncope.

Regarding device therapy for NCS, the devil remains in the details. A 2007 meta-analysis of nine randomized trials comparing pacemaker therapy with medical therapy, usual care, placebo, or different pacing algorithms revealed that permanent pacing decreased the risk of syncopal recurrence in unblinded studies (OR 0.09, CI 0.04-0.22) and studies comparing different pacemaker algorithms (OR 0.04, CI 0.0-0.23); however, in the two available double-blinded trials, there was no reduction in syncope recurrence (OR 0.83, CI 0.41-1.70) [3]. This study underscored the role of response expectancy, i.e., the awareness that a pacemaker was implanted and functioning reduced the risk of recurrent syncope (OR $0.16, \mathrm{CI}$ $0.06-0.40, p=0.0001)$. Placebo effects have been observed in both invasive and non-invasive studies and subject response expectancy (patient expects improvement), and/or observer response expectancy (physician expects patient to improve) likely plays an important role in that NCS patients have demonstrated symptomatic improvement even without placebo [4]. This effect was particularly striking in the ISSUE 1 study, which was a prospective observational study in which 111 patients with three or more syncopal episodes in the preceding 2 years in the absence of structural heart disease were implanted with loop recorders. In both the patients with isolated syncope and negative tilt testing and those with tilt-positive syncope, after loop recorder implantation, syncope recurred in only $34 \%$ of patients in the absence of the intervention offering any clinical gain [5]. Similarly, the multicenter, prospective, randomized $\mathrm{PC}$-trial enrolled 223 patients with recurrent vasovagal syncope and randomized them to standardized conventional therapy versus conventional therapy in addition to 
training in physical counterpressure maneuvers [6]. While there was a relative risk reduction of $39 \%$ in the physical counterpressure maneuver group, there was also improvement in syncope burden in the conventional group, which consisted of an explanation of the mechanisms of vasovagal syncope and lifestyle modification advice.

In this issue of JICE, Gunda et al. [7] present the first study to examine the effectiveness of yoga as a treatment for neurocardiogenic syncope in young female patients who do not respond to maximal supportive therapy. This small pilot study enrolled 44 patients, each of whom received selfdirected yoga instruction on a DVD. For the 21 patients who actually practiced yoga as directed, they experienced a statistically significant improvement in the number of syncopal and presyncopal episodes, decrease in positive head-up tilt table response, as well as a decrease in the Syncope Functional Status Questionnaire Score from enrollment to study completion.

This small pilot study has severe limitations in that the patient selection is not necessarily representative of the wider population of NCS patients; the frequency of syncope/ presyncope during the control phase of the study was very high, itself predicting recurrence; and the study design with intervention phase following control phase may have created bias. Although this study represents just a small subset of patients suffering from NCS, it poses an interesting alternative - or as the authors suggest - adjunctive therapy for patients with recurrent NCS who have failed traditional pharmacologic and maximal supportive therapies.

Because NCS does not affect survival, treatment should focus on improving symptoms and optimizing quality of life. Often, syncope recurrence decreases over time even in the absence of specific intervention [8]. Given the lack of evidence supporting the effectiveness of traditional pharmacologic and device-based therapy for symptom reduction and improved quality of life in patients with NCS, it is reasonable to consider lifestyle modification-based therapies. Lifestyle recommendations can prove effective interventions in that they require adherence, which in itself can promote a placebo and expectancy response in addition to a salutary effect of the training itself [9]. As an ancient form of exercise including breathing exercises, meditation, and isotonic exercise in a variety of postures, yoga may offer an innovative, low-risk, costeffective lifestyle intervention with no significant side effects. We look forward to the authors' planned larger, prospective, randomized clinical trial, which may enlighten us all.

\section{References}

1. Romme, J.J., Reitsma, J.B., Black, C.N., Colman, N., Scholten, R.J., Wieling, W., VanDijk, N. (2011). Drugs and pacemakers for vasovagal, carotid sinus and situational syncope. Cochrane Database of Systematic Reviews, 5(10), CD004194.

2. Vyas, A., Swaminathan, P. D., Zimmerman, M. B., \& Olshansky, B. (2013). Are treatments for vasovagal syncope effective? A metaanalysis. International Journal of Cardiology, 167, 1906-1911.

3. Sud, S., Massel, D., Klein, G. J., Leong-Sit, P., Yee, R., Skanes, A. C., Gula, L. J., \& Krahn, A. D. (2007). The expectation effect and cardiac pacing for refractory vasovagal syncope. American Journal of Medicine, 120(1), 54-62.

4. Inderjeet, S., Sheldon, R., \& Pournazari, P. (2014). Clinical improvement of vasovagal syncope in the absence of specific therapies: the Seinfield effect. Cardiology Journal, 21(6), 637-642.

5. Moya, A., Brignole, M., Menozzi, C., Garcia-Civera, R., Tognarini, S., Mont, L., Botto, G., Giada, F., Cornacchia, D., \& International Study on Syncope of Uncertain Etiology (ISSUE) Investigators. (2001). Mechanism of syncope in patients with isolated syncope and in patients with tilt-positive syncope. Circulation, 104(11), 1261-1267.

6. van Dijk, N., Quartieri, F., Blanc, J. J., Garcia-Civera, R., Brignole, M., Moya, A., Wieling, W., \& PC-Trial Investigators. (2006). Effectiveness of physical counterpressure maneuvers in preventing vasovagal syncope: the Physical Counterpressure Manoeuvers Trial (PC-Trial). Journal of the American College of Cardiology, 48(8), 1652-1657.

7. Gunda S, Kanmanthareddy A, Atkins D, Bommana S, Pimentel R, Drisko J, Dibiase L, Beheiry S, Hao S, Natale A, Lakkireddy D. Role of yoga as an adjunctive therapy in patients with neurocardiogenic syncope: a pilot study. Journal of Interventional Cardiac Electrophysiology. 2015 Apr 12. PMID: 25863799. [Epub ahead of print]

8. Natale, A., Geiger, M. J., Maglio, C., Newby, K. H., Dhala, A., Akhtar, M., \& Sra, J. (1996). Recurrence of neurocardiogenic syncope without pharmacologic interventions. American Journal of Cardiology, 77(11), 1001-1003.

9. Hawkins, N. M., \& Krahn, A. D. (2014). Does any therapy really work for neurocardiogenic syncope? Cardiology Journal, 21(6), 616-624. 